SECTION 5. Innovative technologies in science.

Tkachenko Irina Mikhailovna

Candidate of Technical Sciences, associate professor

Yuri Gagarin State Technical University of Saratov, Russia tkachenko-i-m@yandex.ru

Zakharov Alexander Alexandrovich

Doctor of Engineering, professor

Yuri Gagarin State Technical University of Saratov, Russia zaharov@sstu.ru

Kozhanova Eugene Romanovna

Candidate of Technical Sciences, associate professor

Institute Technicians Technologics and Managements of Balakovo, Russia ljubim@,bk.ru

\title{
STUDY OF THE EFFECT OF PRECESSION OF THE SPIN STRUCTURE AS AN ELEMENT RECOGNITION SYSTEM WITH THE USE OF NEURAL NETWORKS
}

\begin{abstract}
The work of complex structures recognition matrix type with a specific task in different branches of science, can be built with the help of the theory of neural networks, and in most cases these systems belong to a heterogeneous type. If this describes a simple dichotomous structure type with the use of forward-propagation neural network - layer perceptron. Considered the effect of precession as neural pattern recognition, using the fundamentals of spintronics.
\end{abstract}

Key words: neural network, recognition system, neuron, spin, precession effect.

\section{ИССЛЕДОВАНИЕ ЭФФЕКТА ПРЕЦЕССИИ СПИНОВОЙ СТРУКТУРЫ КАК ЭЛЕМЕНТА СИСТЕМЫ РАСПОЗНАВАНИЯ С ИСПОЛЬЗОВАНИЕМ АППАРАТА НЕЙРОННЫХ СЕТЕЙ}

\begin{abstract}
Аннотация: Работа сложных структур распознавания матричного типа $c$ определенной поставленной задачей в различных отраслях науки, может быть алгоритмизирована с помощьью теории нейронных сетей, при этом в большинстве случаев такие системы принадлежат к неоднородному типу. При этом описана простейшая структура дихотомического типа с использованием нейронной сети прямого распространения - однослойный персептрон. Рассмотрен эффект прецессии как нейронная модель распознавания образов, используя основы спинтроники.
\end{abstract}

Ключевые слова: нейронная сеть, система распознавания, нейрон, спин, эффект прецессии.

Нейронная сеть представляет собой совокупность большого числа сравнительно простых элементов - нейронов, топология соединений которых зависит от типа сети. Чтобы создать нейронную сеть для решения какой-либо конкретной задачи, мы должны выбрать, каким образом следует соединять нейроны друг с другом, и соответствующим образом подобрать значения весовых параметров на этих связях. Может ли влиять один элемент на другой, зависит от установленных соединений. Вес соединения определяет силу влияния $[4 ; 5$, с. 12$]$.

Работа сложных структур распознавания матричного типа с определенной поставленной задачей в различных отраслях науки, могут быть алгоритмизированы с помощью теории нейронных сетей, при этом в большинстве случаев такие системы принадлежат к неоднородному типу. Выбор структуры нейронной сети определяется сложностью модели матричного типа, отображающей сложность решаемой задачи 
идентификации элементов системы. Для решения оптимальных задач уже существует ряд конфигураций. Рассмотрим пример описания простейшей структуры дихотомического типа с использованием нейронной сети прямого распространения однослойный персептрон [1]. При этом один входной образ может описывать два класса, каждый соответствующий логическому уровню «0» или «1». Для повышения достоверности лучше, когда каждому классу соответствует отдельный элемент системы - нейрон (образ, спин и т.д.). На $\mathrm{n}$ входы однотипных, принадлежащих одной категории, элементов основного множества - нейронов, подаются сигналы, в виде информационного потока, проходящие по синапсам на 4 нейрона, образующие один слой нейронной сети и выдающие выходные сигналы (рис. 1a). В зависимости от уровня активации (соответствия) входного сигнала, который определяется функцией активации F, на выходе будет либо логическая единица, либо логический ноль.

Вход 1

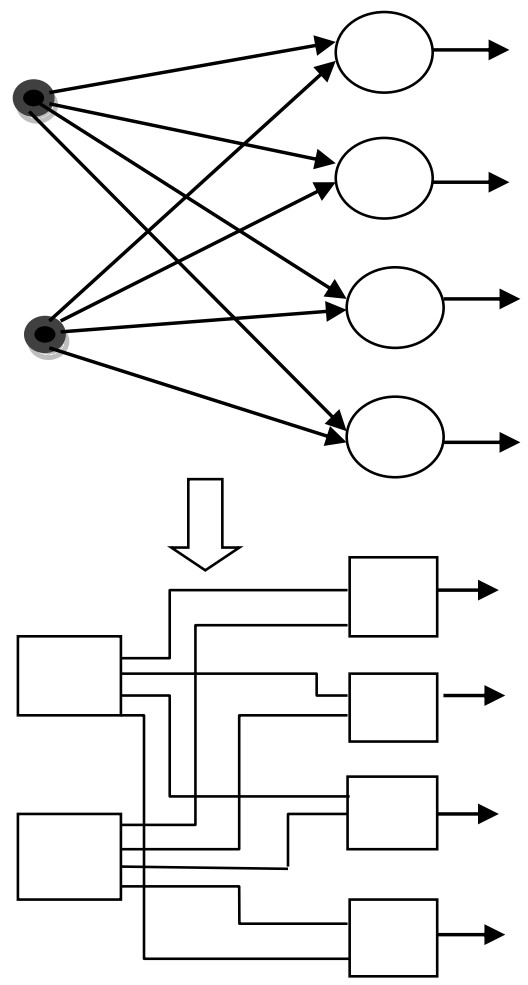

Вход 1
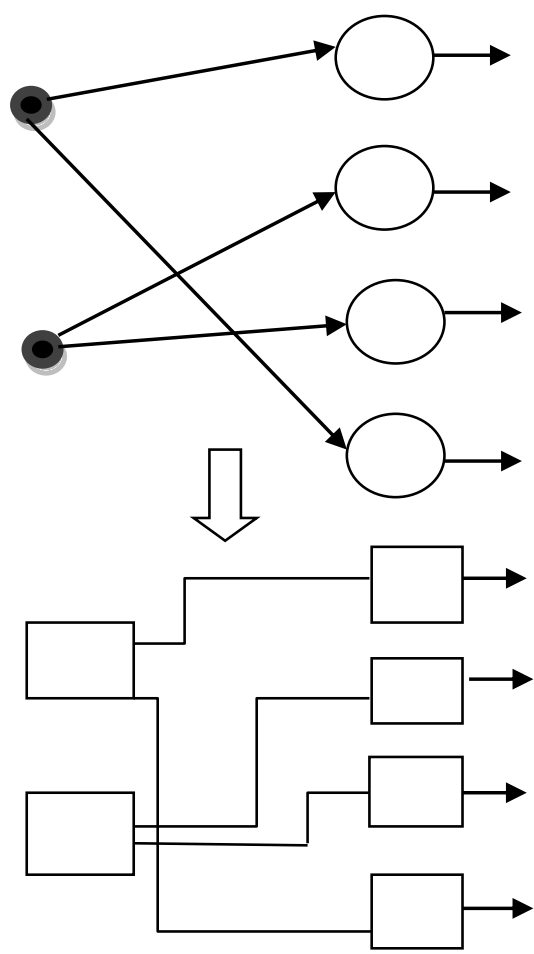

Рисунок 1 - Матричная структура как нейронная сеть прямого распространения.

При построении нейронных сетей, учитывая все особенности задачи, можно убрать синапсы, обладающие неинформативными потоками, где функция активации равна нулю. (рис.1б) Это может быть использовано в тестовых технологиях при построении логистических моделей композиций матричного типа с целью их оптимизации $[2 ; 3 ; 7]$.

Рассмотрим эффект прецессии как нейронную модель распознавания образов матричного типа, используя основы спинтроники. В теории магнетизма считается, что электрон обладает квантовым свойством - спином, вращающейся вокруг своей оси и соединяющей южный и северный полюса электрона. Спины электронов могут быть ориентированы в направлениях, которые обычно называют "спин-вверх" и "спин-вниз". (рис 2). 

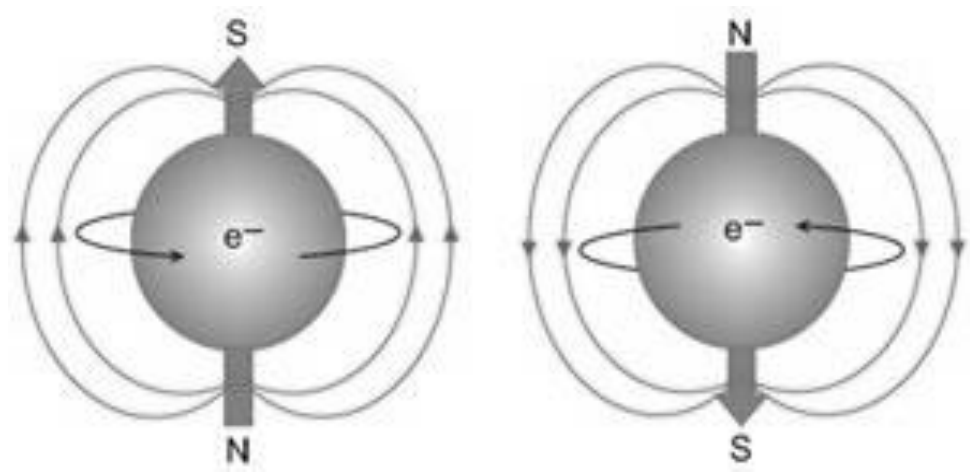

Рисунок 2 - Спин электрона.

Если поместить электроны в магнитное поле, то их спины выстроятся вдоль направления поля. Если выключить поле, прецессия спина прекращается и его ориентация фиксируется. Другими словами, используя эффект прецессии, можно менять спиновое состояние электрона и тем самым изменять бит информации, переносимый электроном, с логического "0" на "1" и обратно, изменяя бит информации. Данный процесс лежит в основе одного из перспективного направления спинтроники, в основе устройства - спиновая память, реализация которой позволяет перейти от битов к так называемым фитам, фазовым числам, способным принимать больше значений, что соответствует большей плотности записи информации. С точки зрения теории нейронных сетей спин электрона представляет собой нейрон, а функцией активации в таких спиновых системах выступает наличие или отсутствие магнитного поля, действие которого вызывает возбуждение или торможение спина. Работа многослойных спиновых структур может быть описана с помощью многослойной сети прямого распространения - многослойный персептрон [5, с.12].

Процессы, протекающие в спиновой структуре, могут быть исследованы с использованием модели технического нейрона МакКаллока и Питтса, основанной на использовании бинарного порогового элемента. Он вычисляет взвешенную сумму $\mathrm{n}$ входных сигналов и формирует на выходе сигнал величины «1», если эта сумма превышает определенный порог $\mathrm{U}$, и «0»- в противном случае, пороговая функция такой модели может быть описана с использованием метода вейвлет-преобразования с помощью вейвлета Хаара $[8 ; 9 ; 10]$. Положительные веса соответствуют возбуждающим связям, а отрицательные - тормозным. При соответствующим образом подобранных весах совокупность параллельно функционирующих нейронов подобного типа способна выполнять универсальные вычисления [5, с.12; 6, с.5].

Таким образом, одной из задач, решаемой интеллектуальной нейронной сетью, является «Управление». При этом в системах управления с эталонной моделью целью управления является расчет такого входного воздействия, при котором система следует по желанной траектории, диктуемой эталонной моделью. В выше описанном примере такая траектория представляет собой процесс передачи информации, который заключается в изменении спинового состояния электрона и тем самым бита информации, с логического "0" на "1" и обратно, что лежит в основе развития такого направления , как спиновая память и т. д.

\section{ЛИТЕРАТУРА:}

1. Ткаченко И.М., Захаров А.А., Кожанова Е.Р. Персептрон как структурный элемент системы распознавания образов // Актуальные проблемы современной науки: сборник статей Междунар. Науч. - практ. конф. Уфа: РИЦ БашГУ. - 2013. Ч.4 - С. 248 -251 . 
2. Ткаченко И.М., Захаров А.А.Модель проектирования тестовых структур на основе текстов различного уровня // Актуальные проблемы электронного приборостроения АПЭП - 2010: материалы Междунар. науч. - техн. конф. Саратов: СГТУ. - 2010. - С.343-347.

3. Ткаченко И.М. Захаров А.А., Калашникова Е.Н. Проектирование моделей тестовых структур на основе логистических схем с произвольным числом множеств и элементов // Саратов: Вестник Саратовский государственный технический университет, 2009.№2(39) Вып.2 с.92-101.

4. Барский А. Б. Нейронные сети: распознавание, управление, принятие решений. - М.: Финансы и статистика, 2004. - 176 с.

5. Комашинский В.И., Смирнов Д.А. Нейронные сети и их применение в системах управления и связи. -М.: Горячая линия-Телеком, 2003. -94с.

6. Аксенов С.В., Новосельцев В.Б. Организация и использование нейронных сетей (методы и технологии) / Под общ. ред. В.Б. Новосельцева. - Томск: Изд-во НТЛ, 2006. -128 c.

7. Захаров А.А. Измерительные системы в профессиональном образовании на основе тестовых технологий. / А.А. Захаров - Саратов: Сарат. гос. техн. ун-т, 2003.$200 \mathrm{c}$.

8. Захаров А.А., Кожанова Е.Р. Применение вейвлет - преобразований для анализа дихотомического сигнала // Вестник СГТУ, 2009 - № 3 (40) Выпуск 1. - С. 59 65.

9. Кожанова Е.Р., Захаров А.А. Возможность применения вейвлет-функций для анализа дихотомического сигнала на примере системы отбраковки и настройки магнитной системы // Йошкар-Ола: Новый университет. - № 1(7), 2012. - С.29-32.

10. Кожанова Е.Р., Захаров А.А., Ткаченко И.М. Сравнительные характеристики применения классического преобразования Фурье и непрерывного вейвлетпреобразования для анализа сигналов. / Е.Р. Кожанова, А.А. Захаров, И.М. Ткаченко Саратов, Сарат. гос. техн. ун-т, 2012, - 132 с. 